\title{
АЛГОРИТМ ВЫБОРА ОПТИМАЛЬНОГО МЕТОДА ОЦЕНКИ ЭЛЕМЕНТОВ ОТЧЕТНОСТИ И СРАВНЕНИЕ ЕГО РЕАЛИЗАЦИИ В РОССИЙСКИХ И ЗАРУБЕЖНЫХ КОМПАНИЯХ
}

\author{
(c) 2021 Демина Ирина Дмитриевна \\ доктор экономических наук, профессор \\ профессор Департамента аудита и корпоративной отчетности \\ Факультета налогов, аудита и бизнес-анализа \\ Финансовый университет при Правительстве Российской Федерации, Россия, Москва \\ (c) 2021 Рудович Эвита Сергеевна \\ студент Департамента аудита и корпоративной отчетности \\ Факультета налогов, аудита и бизнес-анализа \\ Финансовый университет при Правительстве Российской Федерации, Россия, Москва
}

В статье рассмотрены особенности подходов к выбору баз оценки. Разъяснены отличия между применением различных методов оценки в российском и зарубежном учете. Результаты исследования показывают необходимость усовершенствования системы контроля за достоверностью финансовой отчетности.

Ключевые слова: базы оценки, достоверность, качественные характеристики отчетности, справедливая стоимость, переоценка, правдивое представление, сопоставимость.

Оценка, несомненно, является одной из наиболее неоднозначных сфер учета, основная сложность которой заключается в выборе метода оценки, который бы отражал финансовую информацию наиболее достоверным образом. Ни концептуальные основы МСФО, ни РСБУ не дают четкого последовательного алгоритма, позволяющего выбрать правильную базу оценки - очевидно, по причине субъективности и уникальности каждого экономического события, требующего оценки. На основе рекомендаций относительно выбора баз оценки, данных в концептуальных основах [1], была составлена модель выбора наиболее подходящего метода оценки, представленная на Рисунке 1. За основу алгоритма выбора были взяты основополагающие качественные характеристики: уместность и правдивое представление (достоверность), так как максимальное повышение уровня данных свойств информации в отчетности является одним из основных принципов качественной отчетности. Стоит также отметить, что вполне допустимы случаи противоречия, в которых сохранение одной качественной характеристики означает отказ от другой - например, компания, владеющая активом, ставит основной целью данного актива его продажу, что предполагает использование текущей оценки, но при этом активный рынок данного вида актива отсутствует, что затрудняет процесс формирования текущей оценки. В таких случаях стоит подходить индивидуально к каждому случаю и принимать решение о том, какой аспект достоверности более важен в конкретной ситуации, а также какой вариант будет менее затратным. Говоря о затратности, ограничение в виде затрат на осуществление того или иного типа оценки является таким же значимым фактором, как и качественные характеристики. К примеру, в некоторых случаях текущую оценку сложно сформировать ввиду отсутствия активного рынка, а затраты на экспертную оценку значительно превышают необходимость предельно точной и достоверной оценки. В таких случаях компания должна задействовать профессиональное суждение и сформировать наиболее целесообразный подход к оценке.

Для того, чтобы выяснить, в какой степени каждая из приведенных на Рисунке 1 выполняется при составлении отчетности был проведен сравнительный анализ по выборке из 10 отчетностей компаний -5 российских, составленных по РСБУ и 5 зарубежных, составленных по МСФО. Сравнение подходов к выбору метода оценки в аналогичных ситуациях позволит сделать выводы о том, какие стандарты более приспособлены для отражения финансовой информации наиболее достоверным способом и какие меры 


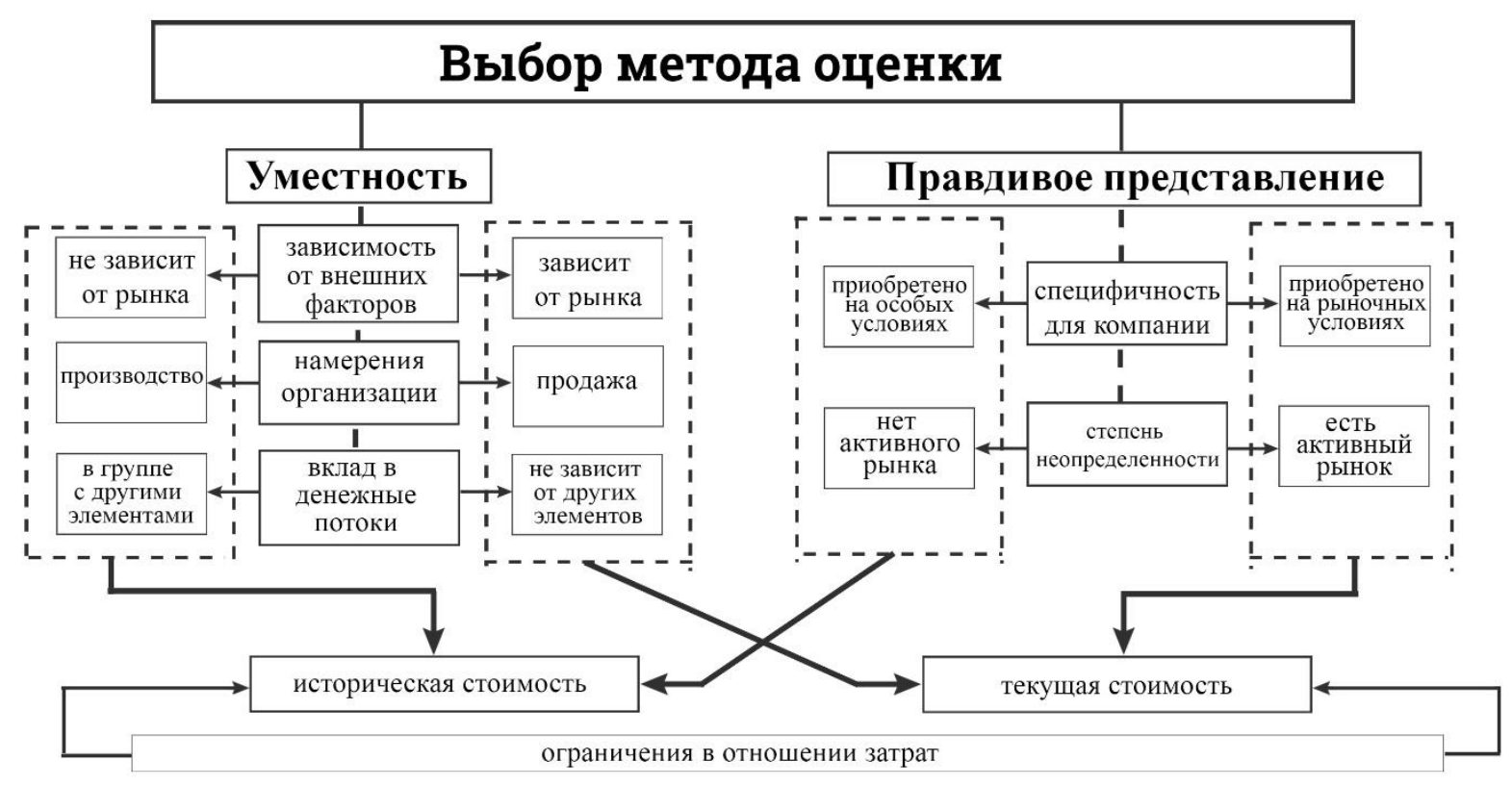

Рисунок 1. Модель выбора базы оценки, основанная на качественных характеристиках Источник: составлено автором

следует предпринять для повышения уровня достоверности в отчетности российских компаний.

Как показано на Рисунке 6, каждая основополагающая качественная характеристика проявляется в разных аспектах оцениваемых элементов. Уместность той или иной базы оценки подразумевает отражение всех особенностей оцениваемого элемента.

Первым таким примером является зависимость актива или обязательства от рыночных факторов. Очевидно, что элементы отчетности, в значительной мере подверженные влиянию рынка наилучшим образом будут представлены текущей стоимостью - например, такая ситуация применима к производным финансовым инструментов, стоимость которых является крайне волатильной. В таких случаях историческая стоимость может существенно отличаться от текущей, тем самым, снижая достоверность информации. Данный аспект был полностью учтен во всех 5 анализируемых зарубежных компаниях - все они оценивают производные финансовые инструменты по справедливой стоимости, максимально приближая отчетность к реальности. Отдельно стоит выделить отчетность Singapore Airlines Ltd [2] - в связи с тем, что компания использует большое количество финансовых инструментов, таких как опционы и фьючерсы для хеджирования цен на топливо, а также с тем, что значительная часть доходов подвержена влиянию курсовых рисков, компа- ния предоставляет детальный анализ всех активов, подверженных рыночным колебаниям. В примечаниях к отчетности показана методика оценки таких активов, а также приведен анализ рисков и степени их влияния на общий финансовый результат. Такой подход к оценке значительно повышает достоверность, однако уместен только в тех компаниях, у которых рыночным рискам подвергаются существенная часть активов. Среди российских компаний только одна использует текущую стоимость для оценки производных финансовых инструментов - ПАО «Аэрофлот» [3], однако стоит отметить, что в остальных компаниях доля данного типа активов либо вообще отсутствует, либо крайне мала, также присутствуют резервы под обесценение. Таким образом, применение справедливой стоимости более распространено среди зарубежных компаний, составляющих свою отчетность по МСФО, вследствие чего их отчетность может быть более достоверна.

Вторым аспектом выбора метода оценки, рассмотренным на Рисунке 1 , является ее соответствие намерениям организации в отношении данного актива или обязательства. К примеру, если компания намеревается продать актив, очевидно, что оценка по текущей рыночной стоимости наилучшим образом отразит выгоды, которые планирует получить компания от данного актива. И наоборот, если компания планирует использовать актив в производстве на 
протяжении всего полезного срока его службы, отражение в балансе исторической стоимости за вычетом амортизации и убытков от обесценения будет более уместно. Анализ рассматриваемых компаний показал, что как российские, так и зарубежные компании в основном придерживаются такого принципа. Большинство рассмотренных компаний оценивает свои основные средства по амортизированной стоимости. У некоторых из них, например ПАО «Аэрофлот» или Singapore Airlines Ltd, имеется незначительное количество ос, которые предназначены для продажи, однако и они оцениваются по амортизированной стоимости, так как польза от сопоставимости, которую обеспечивает такой подход, превышает пользу от более точной оценки по рыночной стоимости. Стоит также отметить, что зарубежные компании гораздо чаще проводят переоценку - раз в год в то время, как компании, составляющие отчетность по РСБУ либо вообще не проводят переоценку, либо проводят крайне редко - например, ПАО «Газпром» последний раз проводило переоценку основных средств (за исключением зданий) в 2016 году [4]. Отдельно стоит отметить подходы к выбору метода амортизации компании «Royal Dutch Shell» [5], которая амортизирует ОС, относящиеся к производству углеводородов пропорционально объему изготовленной продукции по всем подтвержденным источникам сырья на месторождении, на котором применяются данные OC - такой подход позволяет лучше отразить намерения организации относительно данных активов и значительно повышает достоверность. Однако, для расчета по такому методу, оценка стоимости подтвержденного объема находящихся на месторождении полезных ископаемых производится по средневзвешенной рыночной цене на соответствующие полезные ископаемые, что может привести к значительным колебаниям данного значения, связанного с нестабильностью рыночных цен на нефть и газ. Это может привести к искажению суммы амортизации и менее достоверному отражению стоимости рассматриваемых активов. Поэтому для поддержания суммы амортизации в более стабильном состоянии, компания производит оценку основываясь на самостоятельном расчете прогнозных показателей цен на нефть и газ, чтобы более точно отразить будущие экономические выгоды от данных ОС. Это является примером того, как используя профессиональное суждение компания смогла существенно повысить достоверность финансовой информации в отчетности. Примером сомнительного способа оценки является оценка основных средств компании «Network Rail» [6]. Данная компания оценивает железнодорожные пути и составы, используемые в основной деятельности, по справедливой стоимости, что создает несоответствие между методом оценки и способом, которым данные ОС будут приносить экономические выгоды.

Третьим рассмотренным фактором, обеспечивающим повышение уместности информации в отчете, является формирование оценки в зависимости от того, каким образом оцениваемые активы формируют денежные потоки. В качестве примера можно привести ситуацию, когда группа активов взаимосвязаны друг с другом и каждый из них является неотъемлемой частью одного производственного процесса. В таком случае применение одинаковых подходов к оценке в рамках одной группы активов позволит повысить уместность, а также понятность отчетности. Примером компании, в которой применяется такой принцип является ПАО «Газпром» - компания отдельно выделяет группу основных средств «Здания», по которой переоценка проводится реже, чем по остальным активам. Также, компании «Network Rail» и «Poстелеком» [7] выделяют среди активов сложные объекты основных средств - комплекс основных средств, которые имеют общее назначение и работают зависимо друг от друга. В отчетности такие объекты учитываются как один объект основных средств, что делает отчетность более понятной.

Три предыдущие характеристики элементов отчетности обеспечивают уместность финансовой информации, полученной в результате оценки. Отдельно также стоит рассмотреть, что обеспечивает ее достоверность. В первую очередь, это степень отражения специфики той или иной сделки. Например, в случае если ввиду особенностей компании она может получить актив или обязательство по цене, значительно отличающейся от рыночной, более уместно будет использование стоимости замещения/исполнения или исторической стоимости. Например, нематериальные активы в большинстве случаев создаются с учетом специфики конкретной организации и труднореализуемы вследствие отсутствия активного рынка. Такие активы проще и целесообразнее оценивать по исторической 
стоимости или стоимости замещения. Действительно, практически все из рассмотренных компаний оценивают НМА по исторической стоимости. Некоторые компании еще больше подстраивают методы оценки под особенности своей деятельности - ПАО «Газпром», например, оценивает поисковые нематериальные активы, такие как лицензии на добычу полезных ископаемых, по стоимости замещения, однако не начинает начислять амортизацию пока не будет подтверждена коммерческая целесообразность добычи. Таким образом, в плане соотнесения особенностей компании, которые существенно влияют на стоимость активов или обязательств, и метода оценки, как российские, так и зарубежные компании показали достаточный уровень достоверности. Примером компании, которая нарушает рассматриваемый подход, является «Vodafone» [8], которая оценивает часть нематериальных активов, специфичных для организации, в частности, клиентской базы и бренда, по справедливой стоимости. В отчете написано, что при оценке используются рыночные цены, однако учитывая отсутствие активного рынка на подобные активы, можно предположить, что формируемая оценка недостаточно достоверна.

Bсе предыдущие рекомендации следует также рассматривать с учетом уровня неопределенности, который несет тот или иной метод оценки. В частности, чаще всего с неопределенностью связано применение метода оценки по справедливой стоимости, поэтому очень важно сбалансировать выгоды и риски, которые могут возникнуть. Рассматривая отчетности с точки зрения неопределенности, все рассмотренные отчетности, составленные на основе РСБУ показывают очень низкий уровень неопределенности. Это является следствием того, что российские компании предпочитают оценку по исторической стоимости и ограничено используют профессиональные суждения и оценку по справедливой стоимости. Несмотря на то, что таким образом они не в полной мере используют возможности для повышения достоверности отчетности, это снижает неопределенность. Рассматриваемые компании, составляющие отчетность в соответствии с МСФО, как уже было отмечено ранее, в большей мере используют справедливую оценку для оценки. Однако при этом, почти все из анализируемых компаний создают резервы под изменение справедливой стоимости, тем самым нивелируя неопре- деленность, возникающую при формировании справедливой оценки. Но несмотря на это, все компании уделяют внимание снижению рисков неопределенности в разной мере. Единственной из рассмотренных зарубежных компаний, уделяющая достаточное внимание рискам неопределенности является «Singapore Airlines», которая представляет в годовом отчете детальный анализ всех рисков, в том числе связанных с оценкой по справедливой стоимости и курсовыми разницами. Остальные компании признают резервы только когда есть подтверждение гарантированного появления обязательства в будущих периодах, и не применяют прогнозные модели для оценки рисков. Наибольшему риску неопределенности подвергается компания «Network rail», которая оценивает значительную часть основных средств, в частности, железнодорожные пути и инфраструктуру, по справедливой стоимости. Для формирования справедливой стоимости компания применяет не цены на активно рынке - метод оценки 1 уровня - а прогнозы денежных потоков, которые компания намеревается получить от данных активов. С одной стороны, при правильном расчете, такой метод оценки может представлять намного более достоверную информацию, чем оценка по амортизированной стоимости. Однако он также существенно повышает риски неопределенности и возможность мошеннических действий, так как оценка полностью зависит от профессионального суждения и может подвергаться манипуляциям. Еще одной компанией с высокими рисками неопределенности является «Vodafone». Как уже упоминалось, данная компания оценивает часть нематериальных активов по рыночной стоимости - клиентскую базу и стоимость бренда. Очевидно, что такая оценка формируется в основном за счет профессионального суждения, так как активного рынка для таких специфичных для конкретной компании активов не существует. Однако в отчетности не раскрываются подробности методики оценки, что вызывает сомнения в ее достоверности.

Результаты анализа, описанного выше представлены в Таблице 1 , на основе которой можно сделать выводы о методах повышения достоверности отчетности при оценке активов и обязательств компании.

В общем, в результате проведенного сравнения можно отметить, что российские компании используют оценку по справедливой стоимости 
Таблица 1. Результаты сравнительного анализа компаний на соответствие качественным характеристикам при оценке элементов отчетности

\begin{tabular}{|c|c|c|c|c|c|}
\hline & \multicolumn{3}{|c|}{ Уместность } & \multicolumn{2}{|c|}{ Достоверность } \\
\hline & $\begin{array}{c}\text { учитывает } \\
\text { влияние рынка }\end{array}$ & $\begin{array}{c}\text { соотносится } \\
\text { с намерениями } \\
\text { организации }\end{array}$ & $\begin{array}{c}\text { отражает } \\
\text { связь с другими } \\
\text { элементами }\end{array}$ & $\begin{array}{c}\text { учитывает } \\
\text { особенности } \\
\text { компании }\end{array}$ & $\begin{array}{c}\text { минимизирует } \\
\text { неопределен- } \\
\text { ность }\end{array}$ \\
\hline ПАО «Аэрофлот» & + & + & Н/Д & + & + \\
\hline $\begin{array}{l}\text { Singapore Airlines } \\
\text { Ltd }\end{array}$ & + & + & Н/Д & + & + \\
\hline ПАО «КАМАЗ» & Н/Д & + & Н/Д & + & + \\
\hline Liebherr AG & + & + & Н/Д & + & - \\
\hline ПАО «Газпром» & - & + & + & + & + \\
\hline Royal Dutch Shell Plc & + & + & Н/Д & + & - \\
\hline ОАО «РЖД» & - & + & Н/Д & + & + \\
\hline Network Rail Ltd & + & - & + & + & - \\
\hline ПАО «Ростелеком» & Н/Д & + & + & + & + \\
\hline Vodafone Plc & + & + & Н/Д & - & - \\
\hline
\end{tabular}

Источник: Составлено автором

намного реже, чем зарубежные. По этой причине отчетность российских компаний может не так точно отражать реальную стоимость активов и обязательств. Однако, с другой стороны, зарубежные компании в большей мере подвергаются риску неопределенности, который вызван активным применением профессионального суждения, вследствие чего возникает неоднозначность оценки некоторых элементов отчетности. Еще одним существенным отличием является частота проведения переоценки: компании, составляющие отчетность по МСФО, переоценивают активы не реже одного раза в год, в то время как значительное количество российских компаний вообще не проводят переоценку, а те, которые проводят, делают это крайне редко - раз в 5-7 лет. Таким образом, как в российской, так и в международной системе учета есть свои проблемы при оценке. Однако, учитывая разнообразие и различие подходов к оценке в компаниях из разных отраслей, можно сделать вывод о том, что невозможно четко ограничить выбор организации или разработать универсальный алгоритм в отношении выбора метода оценки. В любом случае остается некоторая степень неопределенности, так как решение о выборе метода оценки основывается на массе факторов, значимость которых варьируется в зависимости от внешних и внутренних условий. Из этого следует, что для повышения уровня достоверности отчетности следует усовершенствовать систему контроля за процессом оценки в организациях.

\section{Библиографический список}

1. Концептуальные основы МСФО 2018 // онлайн-ресурс «Финансовый учет» URL: https://fin-accounting.ru/ ifrs/ifrs-framework

2. Annual financial report for 2020 // Singapore Airlines official website URL: https://www.singaporeair.com/saar5/ pdf/Investor-Relations/Annual-Report/annualreport1920.pdf

3. Годовая отчетость ПАО «Аэрофлот» за 2020 год // Служб раскрытия информации Интерфакс URL: https://edisclosure.ru/portal/files.aspx?id=1480\&type $=3$

4. Годовая отчетость ПАО «Газпром» за 2020 год // Служб раскрытия информации Интерфакс URL: https:// www.e-disclosure.ru/portal/files.aspx?id=934\&type=3

5. Annual financial report for 2020 // Royal Dutch Shell official web site URL: https://reports.shell.com/annualreport/2020/servicepages/downloads/files/shell-annual-report-2020.pdf

6. Annual financial report for 2020 // Network Rail official web site URL: https://www.networkrail.co.uk/wp-content/ uploads/2020/07/Annual-report-and-accounts-2020.pdf

7. Годовая отчетость ПАО «Ростелеком» за 2020 год // Служб раскрытия информации Интерфакс URL: https:// e-disclosure.ru/portal/files.aspx?id=141 \&type=3

8. Annual financial report for 2020 // Vodafone official web site URL: https://investors.vodafone.com/sites/vodafoneir/files/vodafone/annual-report/vodafone-annual-report-2020.pdf 
9. Годовая отчетость ПАО «КАMАЗ» за 2020 год // Служб раскрытия информации Интерфакс URL: https:/edisclosure.ru/portal/files.aspx?id=33\&type=3

10. Annual financial report for 2020 // Liebherr official web site URL: https://www.liebherr.com/shared/media/annualreport/annual-report-2020/pdf/liebherr_annual-report_2020_en.pdf

11. Годовая отчетость ОАО «РЖД» за 2020 год // Служб раскрытия информации Интерфакс URL: https://edisclosure.ru/portal/files.aspx?id=4543\&type $=3$ 